\title{
Trauma in patients with spinal ankylosing disorders is often underestimated and frequently overlooked despite the high likelihood of complications
}

\author{
Peter Grunert, MD, and Doniel Drazin, MD, MA \\ College of Medicine, Pacific Northwest University of Health Sciences, Yakima, Washington
}

$\mathrm{W}$ E share the opinion that the data gathered and analyzed in the retrospective single-center study by Schwendner and colleagues are of great importance regarding the care of spinal injuries in patients with ankylosing spinal disorders (ASDs). ${ }^{1}$ ASDs encompass ankylosing spondylitis (AS), diffuse idiopathic skeletal hyperostosis (DISH), and end-stage spondylosis.

From our training together, we remember the 2 patients who presented to the emergency department (ED) with severe back pain after minor trauma. When their simple radiographs were negative for a fracture, both patients were discharged home with conservative treatment recommendations for muscular strain. Both patients returned to the ED a few days later with neurological deficits, specifically, signs of spinal cord injury. Spinal fractures were diagnosed on subsequent CT scans, and both patients were urgently treated surgically.

Since then, both authors have seen many similar cases at other hospitals where an ASD-related spine fracture, hard to see on a simple radiograph, has been overlooked and consequently impacted a patient's outcome. The data gathered in this publication help to better understand the complex nature of these fractures by quantifying and analyzing the diagnosis and treatment of this condition and the associated risks.

In our opinion, several important results of this study should be emphasized.

1) The majority of patients in the published study presented with back pain, while only $25 \%$ had neurological deficits. ${ }^{1}$ In addition, $78 \%$ of patients had only a minor trauma. Lack of a severe trauma history or lack of neurological deficit often leads providers to underestimate the severity of the patient's condition and the need for further imaging that could help diagnose a fracture (ordering a CT scan after obtaining a radiograph, or ordering MRI after ordering CT imaging). Schwendner et al. point out that $25 \%$ of patients did not undergo immediate CT imaging. It should be mentioned that these data were gathered from hospitals in Germany where imaging costs are less relevant than they are in the US.

2) Another problem addressed in this article is that even if appropriate imaging studies are ordered, fractures can still be overlooked; $33 \%$ of fractures were not correctly identified in this study. In the Discussion section, the authors mention that other trauma centers have reported that up to $45 \%$ of their cases had a delay in diagnosis. This difficulty is due to the fact that these fractures often do not show a vertebral body compression component or a translational component that can easily be seen on CT or radiography. CT sometimes demonstrates only very thin fracture lines, which can be missed; MRI, which could demonstrate soft-tissue injuries, is often not ordered if the CT diagnosis was negative for fracture. Overlooking the fracture can lead to secondary injuries such as spinal cord injury due to the mechanics of the fracture. The authors state that $8 \%$ of patients worsened neurologically due to a missed diagnosis.

3) Eighteen percent of patients in this study had surgical complications, mostly in the form of construct failure, despite being treated at an academic high-volume center. Providers included two (cervical spine) to three (thoracolumbar spine) levels above and below the fracture, which generally would be considered a stable construct. The fundamental problem with these injuries from a surgical perspective is the combination of osteopenic bone with an often multisegmented fused spine above and below the fracture, which creates a significant cantilever. Both circumstances create a high risk for screw pullout and subsequent implant failure, which is why longer constructs are required.

4) Seventy-seven percent of patients treated in this study developed further medical issues (mostly cardiovascu- 
lar or pulmonary) due to their comorbidities. Treating providers should be aware of these postoperative risks that are not directly related to the surgical procedure. Patients at risk should, therefore, likely be observed for a longer period in the hospital and not discharged too soon after surgical treatment of the fracture. In some cases, patients with severe fractures might have to be treated conservatively, especially when respiratory and pulmonary comorbidities are present.

Schwendner and colleagues did an excellent job of highlighting the increased problems in the diagnosis, treatment, and risks associated with patients who have an ASD and present in the ED after a trauma, even a minor one, and the need for more thorough imaging of their entire spine. Overlooking a spinal fracture can lead to the high likelihood of complications from the unstable spine. The increased medical risks associated with ASDs, including a higher mortality rate even when there are no surgical complications, need to be considered and explained to the patients and their families.

https://thejns.org/doi/abs/10.3171/2021.7.FOCUS21436

\section{References}

1. Schwendner M, Seule M, Meyer B, Krieg SM. Management of spine fractures in ankylosing spondylitis and diffuse idiopathic skeletal hyperostosis: a challenge. Neurosurg Focus. 2021;51(4):E2 .

\section{Disclosures}

The authors report no conflict of interest.

\section{Correspondence}

Doniel Drazin: ddrazin@gmail.com.

\section{INCLUDE WHEN CITING}

DOI: 10.3171/2021.7.FOCUS21436. 